\title{
Viewers Habits Mining By Combine TV Rating with Intermedia Specify
}

\author{
Bonam Rajiv Kumar, M.Naresh
}

\begin{abstract}
TELEVISION rankings square action a broad previously owned sign within the TV broadcasting area. whereas TELEVISION rankings square measure primarily used in advertising and marketing, they'll even be utilized as a social tool that mirrors the rate of interests of people. This paper presents a framework for situating audience behavior through the mining of TELEVISION rankings. we have actually obtained established a structure that permits exploration of varied patterns of target market behavior from TV scores. Made use of together with various multimedia system components like video clip as well as message, it allows many sort of information to be semi-automatically found, like what kinds of news programs square action of many rate of interest and what square measure the essential visual options for getting high TELEVISION ratings. the innovation of target market practices is attained by that focus on the modification factors within the ranking expertise, i.e., the moments anywhere numerous folks change the network or turn the tv on or off. made descriptions that identify these factors square measure extracted from multimedia system materials, so numerous filtering techniques square measure accustomed essence details patterns of passion. lots of applications of this framework for finding detaincontestable that it will efficiently extract numerous type of target market practices. To the most effective of our data, this job is that the initial job to explore the employment of ratings expertise along with video clip and also different multimedia system expertise.
\end{abstract}

\section{INTRODUCTION}

TV gathering of people analyses (TELEVISION appraisals), which are used to evaluate the popularity of TELEVISION programs, is a key guideline in the field of TELEVISION broadcasting. The TELEVISION score of a program shows the degree of all TV family units listened to that program. Television evaluations are mostly utilized by fans as well as fans to quantify the compass of the backers' promoting. For promoting, Because what number of focused individuals are observing the promotion (i.e., company movies (CFs)) is important to supports, the appraisals are a crucial pen. Telecasters concentrate on broadening the evaluations of their tasks so as to get more customers. Tv assessments can similarly be used as sensor to determine the passions of individuals. In case the substance of a TV program intrigues people, they will certainly as a whole check out the program, and the assessments subsequently increment. At the same time, if individuals are not fascinated

Revised Manuscript Received on July 10, 2019.

Bonam Rajiv Kumar, M.Tech II YEAR,Computer Science And Engineering, Newton's Institute of Engineering, Andhra Pradesh, India

M.Naresh, Assoc Prof, Computer Science And Engineering, Newton's Institute of Engineering, Andhra Pradesh, India by the material, they transform to different channels, and the evaluations decline. Consequently,

TELEVISION evaluations are a pen of mainstream styles and also social patterns, e.g., what type of information are of intrigue likewise, which entertainers are already renowned. Telecasters that find such information by mining TELEVISION evaluations info can make Tv programs that are required by numerous individuals. Albeit social networks locations, as an example, Facebook and twitter are likewise helpful media for catching the interests of people, simply a little component of the customers of such media are dynamic customers, so the interests of the substantial majority i.e., the quiet majority, are ignored.TV appraisals in addition include crucial data for risk the execs. In a situation, for example, a tragic occasion, the government needs to promptly convey appropriate information to individuals in a hassle-free way. Since TELEVISION is a key tool for handing down information to numerous people continuously, exactly how to appropriately communicate essential data by TELEVISION should be thought about. By breaking down TV evaluations to decide what number of people get information from TELEVISION, we can pass judgment on whether standard data has been properly conveyed and also take actions to boost the dispersing of such information. Despite the fact that TV appraisals have actually been checked out for a very long time, the majority of previous work concentrated on guaging the appraisals of details TV programs. The ideas was to appraise the cost of TV advertising in light of the fact that the expenditure of advertising is particularly connected to the TELEVISION evaluations. Hardly any jobs focused on the mining of examinations info despite the fact that such details consists of substantial information. Also, unification of TV evaluations with sight and sound compound, as an example, video details for TV programs has actually not been explored. The consolidation of TELEVISION appraisals with view and also audio substance (e.g., video, discussion) urges locating the connections between team of sightseers practices and TELEVISION program substance.demonstrates the assessments information for two TV station that connect live the flooding of the Kinugawa River on Sep. 10, 2015. Thumbnail photos of the two communicates around the season of the flooding are similarly appeared. Both stations were communicating live up till the factor when the time shown by the red spotted line. By then, one terminal (talked with by the

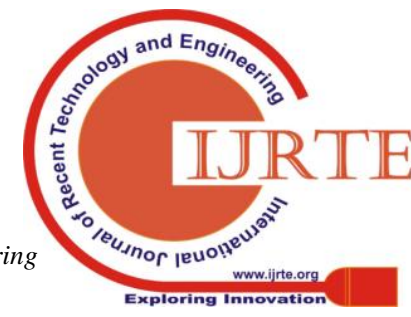


environment-friendly line) began appearing CFs. This triggered a significant modification in spectators from the "environment-friendly" station to the "blue" terminal. Comparative conduct from the "blue" station to the "green" terminal is in addition seen at dark specked line. This implies the watchers were significantly fascinated by the celebration and in this way changed to another station to proceed seeing the news. This is a situation of group of onlookers carry out received from $\mathrm{Tv}$ assessments details for just a solitary event. Examination of bigger amounts of such information will equip normal examples of celebration of individuals carry out to be discovered consequently, and also significantly concealed practices can be discovered by collaborating TV assessments and also media compound. The motivation for discovering team of sightseers conduct has a few perspectives. As an issue of initial importance, celebration of people carry out shows what individuals find of intrigue, and also this understanding is essential for making TV programs that draw in watchers. Recognizing client conduct is also essential from the component of advertising. Obtaining the instances that development assessments from the mining of event of people perform will allow fans to get higher analyses for their jobs as well as in this manner get even more backers. Danger the board is one more angle. Customer conduct mining reveals just how individuals obtain information complying with an ordeal. This information might be used to figure out what should be finished to even more most likely pass on important data to countless people. In this paper, we study the celebrations or instance in group examinations details by utilizing interactive media compound, for instance, video clip information also, records information in mix with evaluations info. Our goal is to accumulate a system that can be made use of to discover the different examples of customer conduct from TV assessments. Despite the fact that client conduct is being investigated by countless works, most of continuous job facility around informal areas and also little job has had a tendency to the conduct of TELEVISION teams of onlookers. To the best of our info, this is the primary service the utilization of event of people assessments accompanied sight and sound details. To discover the links between the examinations information and sight and audio compound, we focus around the adjustment focuses, i.e., the focuses in time when numerous individuals lately have a look at a particular TV program. These concentrates are taken into consideration to have essential information, particularly the interests of the spectators. We show these focuses making use of aesthetic highlights separated from video clip and also catchphrases separated from transcripts. Considering that the variety of such focuses is colossal, we specifically and also adaptably use separating and total based on the investigation target. Tests showed that this system can discover various kind of substantial data. Tv audience rankings (TELEVISION ratings), that make even procedure intend to analyze the recognition of TELEVISION programs, can be a vital sign within the field of TV broadcasting. The $\mathrm{TV}$ rating of a program indicates the proportion of all tv households tuned in to it program. TV scores square action mainly made use of by enrollers and broadcasters to live the reach of the enrollers' advertising. For procedure observance the advertisement( i.e., commercial films( CFs)) is important to enrollers, the ratings square procedure an important sign.

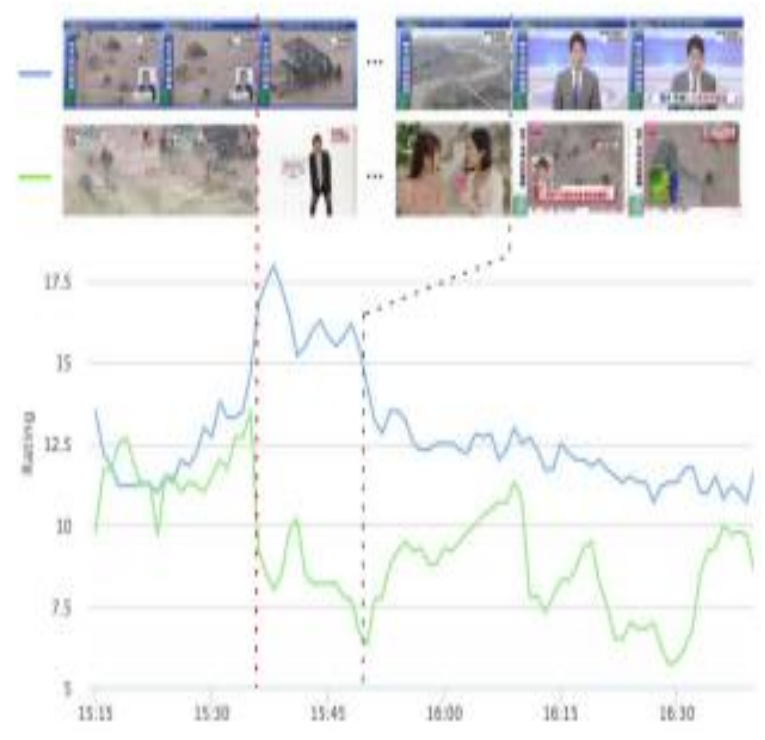

Fig. 1. Audience behaviors observed in TV ratings data at the flooding of the Kinugawa River.

Broadcasters focus on increasing the scores of their programs so as to build up additional sponsors. TV rankings may be utilized as device to determine the rate of interests of individuals. If the components of a program rate of interest individuals, they tend to tune up to the program, and the rankings as a result increase. On the other hand, if folks do not appear to be interested Alternatively, if folks don't seem to be interested regarding the components, they switch over to different networks, and also the scores reduction. Consequently, TELEVISION rankings square measure associate indicator of prevalent subjects and social patterns, e.g., what selections of information square action of passion and that entertainers square step currently prevalent. Broadcasters United Nations agency discover such data by mining TELEVISION rankings details will certainly create TELEVISION programs that settle action required by numerous individuals. though social media sites such as Facebook and twitter location ls houseful media for capturing the passions of individuals, only little section of the customers of such media square procedure active customers, that the passions of the majority i.e., the quiet majority, square step unheeded.Our payments are as complies with: 1) we tend to initiate a research study of mining that targets TV rankings, demonstrating that useful information might be obtained from TV rankings which TELEVISION scores are an important supply as records furthermore due to the fact that the first of check out the mining of TV rankings. 2) we tend to establish a structure for analysing audience behaviour by analysing change factors. It makes use of diverse alternatives as well as filtering strategies presented from different fields like a process visual online. This structure will discover varied type of useful information, as revealed within the

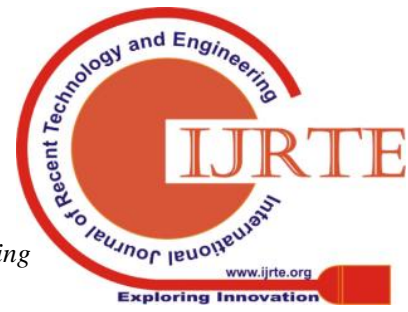


application. given in Sec. VI

\section{RELATED WORK:}

In this paper, we focus on the mining of team of onlookers carry out from TELEVISION assessments info. Our goal is to accumulate a structure for subsequently finding specific examples or occasions showing the interests of people. To this end, we focus around the adjustment focuses in score information. Particularly, we focus around the tiny scale degree change concentrates, where the per-minute evaluations change essentially inside a number of mins, which implies that the amount of spectators increments or diminishes suddenly. It is anticipated that something occurs at these focuses, so the information they provide is much more successful than that offered by different focuses. We determine these concentrates and examine them in mix with video clip compound and various other meta-information such as inscriptions. By studying the substance contrasting to the change concentrates, we can all the more likely understand things that a lot of intrigue spectators. By extracting a substantial variety of development concentrates, we can find the events and also examples revealed by the appraisals details In the first action, we locate nonetheless many change concentrates as can reasonably be anticipated using a simple criterion, relying on later phases to looking them. Our concept center is around stages 2 as well as 3, i.e., representation, dividing, what's even more, combination of development focuses using media material. For each recognized factor, different highlights are divided from one-minute multimedias information at the season of each indicate offer abundant portrayals that explain the focuses. These representation are used to channel as well as complete the concentrates. Separating is made use of to dismiss commotion or focus the focuses that we need to investigate. Finally, the apart focuses are collected what's even more, pictured. For instance, understanding of the adjustment point measurements motivates understanding of assessments increment layout. Customer can wisely add or transform the networks to picture
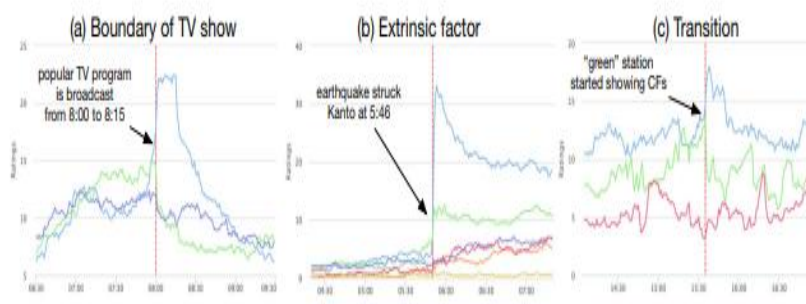

Fig. 2. Examples of three cases observed in audience ratings. (a) Boundary of TV slow: the rating incressed when the programs began. (b) Extrinsic factor. the rating suddenly incresed when an earthquake snuck. (c) Transition: the ratings of two programs suddenly incerased or decreased dox to viewers swiching from one program to the othet.

significantly certain cases, which speeds up the instance revelation. Notwithstanding TELEVISION appraisals info, we utilize a couple of sort of mixed media info to illustrate and transport the modification focuses.: A number of visual highlights are made use of to depict photos as concise in Table I. Shading and also surface are utilized as low-level aesthetic highlights. Product and sensation highlights are made use of as mid-level highlights. These picture highlights figured out for each one of the 60 outlines (one edge for every 2nd) in a one-minute video clip. They are then gathered by taking the mean for every single one over the 60 details, and the collected values are used as the highlights of each change point.Low-level Functions. We use low-level highlights offered from computational feel, which has been seemed powerful in evaluating visual suggestions. Complying with, we actualize the shading as well as surface area highlights made use of in [1]: Color names, GLCM highlights, HSV understandings, and also the Enjoyment, Arousal as well as Strength esteems refined from HSV worths. Mid-level Attributes. Notwithstanding using low-level highlights, we utilize bigger quantity consists of that are progressively related to the image content. We utilize the write-up category order rating as well as the feeling rating specifically. We initially utilize includes based on ImageNet order [2] We register ball game for 1000 post categories based upon the ILSVRC organizing project. We use the AlexNet [3] prepared with the ILSVRC 2012 dataset [2] to figure the organizing ratings (i.e., the probabilities for each classification) and use them as highlights. Since the nature of TELEVISION material contrasts from the categories in ImageNet, it is tough to make use of the scores of distinct 1000 classifications straightforwardly. Rather, we use 2 highlights registered depending on the 1000-class scores. Instantly, we make use of ball games for the 9 top-level courses of ImageNet (plant, land, typical, sporting activity, antiquity, parasite, individual, animal, misc) figured out by accumulating the setup ratings for the 1000 short article classifications. Additionally, we identify item categories reasonable for TELEVISION substance by bunching the highlights of convolutional neural systems. We appreciate the 4096-dimensional fc7 highlights of AlexNet as semantic highlights and do k-implies grouping on them $(\mathrm{k}=100)$ with the objective that each lot talks to specific items or then again scenes that repeatedly turn up in TELEVISION material. Each covering is assigned to the team with closest centroids as well as 100 dimensional paired element are obtained for every case, where each measurement shows whether the casing has a place with each team. Three instances of made numbers (speaking to sumo, various games, and also climate records) are shown up in Figure 4. Regardless of the highlights dependent on the thing course, enthusiastic part is furthermore utilized. we figure the scores for eight feelings (beguilement, outrage, stunningness, joy, sicken, power, worry, dismal) utilizing the technique for Machajdik and Hanbury [1] They showed that sensations stimulated by images can be identified utilizing a computational technique. By using the feeling grouping scores, we can damage down the impacts of the sensations triggered by video clip material on TV assessments. 2)

Keywords: We divide the catch phrases from the subtitle for the moment comparing to

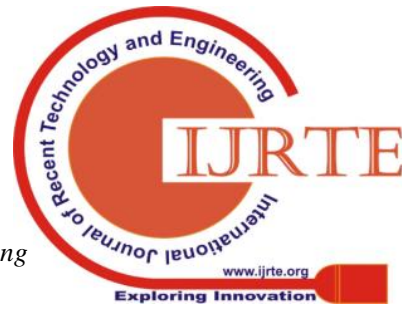


each adjustment factor. To extricate watchwords from Japanese content, we at first execute morphological examination to decay the web content into a few morphemes considering that Japanese web content has no room between words. We do this utilizing the MeCab morphological analyzer [4], which is the normally used for morphological examination of Japanese. It generates the isolated material together with grammatic feature data. As it were things are liberated as confident keywords.Next we decline the stopwords that frequently show up in discussion. In TV compound, there are much of the time showed up words for every Television jobs, as an example, the title of TELEVISION program that are certainly not important to define the compound. To bar these words, we make a model for every one of the $24 \mathrm{hr}$ in a connect duration for every one of the 7 significant TV stations in the Kanto zone additionally, utilize them to establish the word appearance rates. Those words that turn up over $30 \%$ days are disallowed. The words (things) staying are made use of as catch phrases.

\section{EXPERIMENTAL RESULTS \& DISCUSSIONS}

We offer the structure that purposes the examination of news programs. We identify the news accounts of a lot of excitement making use of the setup of progression concentrates. We use increment focuses saw in projects of news and also information program classifications that are required to mirror the watchers' interest to news stories. Investigation of the prevalent news uncovers lucrative knowing. As an example, regardless of whether the objective of TELEVISION stations is at change with the actual interests of individuals can be attested by the connection in between the variety of increment focuses and relaying time. What's more, the testimonial data at catastrophe will certainly discover the cognizance of farce anticipation, which is the necessary details from the part of threat the board We recognize newspaper article making use of catch phrases cooccur with increment focuses. The plan of increment concentrates are deemed the collection of graph centers. To begin with, the centers that are taken into consideration to be a comparable subject are associated, which is determined by catchphrases as well as connect days; 2 factors isolated inside 2 days and having in unwanted of 3 typical catch phrases are associated. Associated parts of graph are after that divided also, every section speaks to the newspaper article. The number of progression concentrates in every newspaper article shows the degree of individuals' excitement for the newspaper article. Top-ten much of the moment showed up catchphrases for each news story are utilized as the signs of the information, which is used to liberate the telecommunications time for every single news. Using this computation, information accounts of typically intrigue can be identified with no earlier details. Although that we attempted some essential bunching computations, for instance, k-implies and also ghostly grouping, they would as a whole union insignificant newspaper article and our straightforward calculation delivered much more specific end results than others. We in addition liberate the telecommunications time for each and every identified news story. Subject department, i.e., finding factor restrictions of news programs, is initial done based on the method utilized in [5] It identifies a subject restriction by discovering a point where the countersign blood circulation has entirely transformed. On the off possibility that a piece incorporates more than 3 of ten catch phrases of a specific newspaper article, we check out the section as that newspaper article. By doing this, we can obtain the telecommunications time of a particular newspaper article if its signs are given. 2) Analysis of News of Rate of interest: We originally assessed whether our strategy can identify necessary newspaper article. We utilized the top-ten news stories in Japan for 2015, as outlined by the Yomiuri Newspaper, as the ground truth. Table II shows the
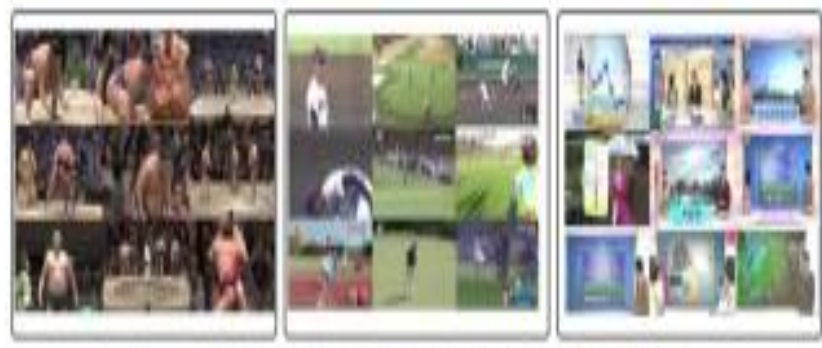

Fig. 4. Examples of clusters created for TV contents.

communication in between the ground fact and our main 10 acknowledgment outcomes. The $\mathrm{CP}$ area demonstrates the rank based on the variety of progress focuses in each identified news. This end result demonstrates that our method identified 5 of ten ground truth. The rank in our outcomes mirrors the dynamic conduct of individuals who want the data about the news, which is unique in relation to the idea of simply essential news. Our outcomes uncovered that the frightening news stories, for example, an instance of homicide or catastrophe will in general do watchers change the channels to proactively gather data of the accounts, while positive news stories, for example, 


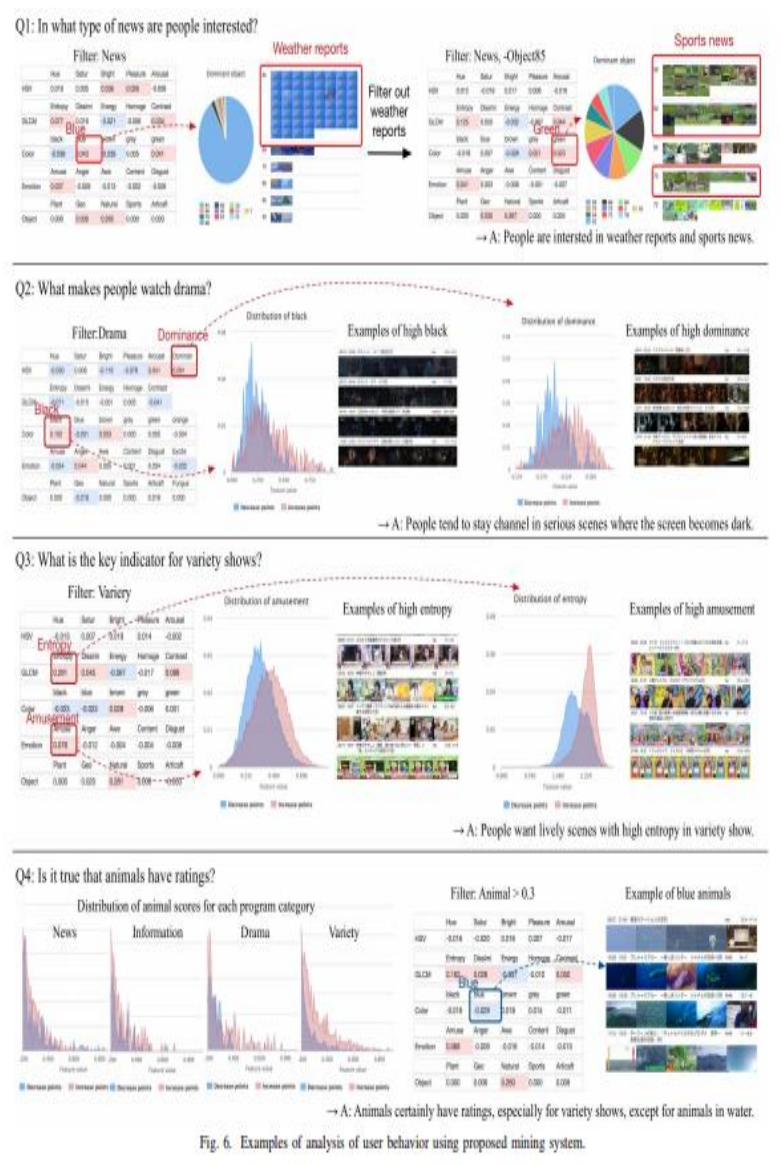

Nobel Prize and Rugby World Cup don't do watchers switch the channels since watchers passivly viewed the tales. We additionally demonstrate the amassed broadcast appointment of every news in Table II (the time segment). The homicide cases that were positioned in our outcome however not positioned in Yomiuri (Osaka, Kawasaki, furthermore, Wakayama cases) were positioned profoundly in broadcast appointment, while the vital news stories that were not recognized by our own for example, the dispatch of Hokuriku Shinkansen were additionally saved much broadcast appointment. It shows TV stations communicate essential news stories similarly to some degree paying little respect to the intrigue of individuals, while news accounts of open intrigue are communicated with some accentuation..

\section{CONCLUSION:}

Our proposed structure incorporating TV appraisals and other sight and sound information such video and content transcripts can be utilized to find group of onlookers conduct. We concentrated on miniaturized scale level change focuses in TV evaluations information that contain profitable data about group of onlookers conduct. A framework dependent on our structure can find the different sorts of learning from a huge number of progress focuses by utilizing different sifting and an accumulation system. The outcomes delivered by our mining instruments exhibited that our system can find various important learning from TV appraisals by consolidating the channels intuitively. Another model application exhibited that framework dependent on our system can identify the news stories of most enthusiasm with no earlier learning.

\section{REFERENCES:}

1. Machajdik, J., Hanbury, A.: Affective image classification using features inspired by psychology and art theory. In: Proc. of ACMMM (2010)

2. Deng, J., Berg, A., Satheesh, S., Su, H., Khosla, A., Fei-Fei, L.: Imagenet large scale visual recognition competition 2012 (ilsvrc2012) (2012)

3. Krizhevsky, A., Sulskever, I., Hinton, G.E.: ImageNet Classification with Deep Convolutional Neural Networks. In: Proc. of NIPS (2012)

4. Kudo, T., Yamamoto, K., Matsumoto, Y.: Applying Conditional Random Fields to Japanese Morphological Analysis. In: Proc. of EMNLP (2004)

5. Ide, I., Mo, H., Katayama, N., Satoh, S.: Topic threading for structuring a large-scale news video archive. In: Proc. of CIVR (2004). 